\title{
Influence of a spatial anisotropy on presence of the intermediate one-half magnetization plateau of a spin-1/2 Ising-Heisenberg branched chain ${ }^{\star}$
}

\author{
Jozef Strečka ${ }^{\mathrm{a}, *}$, Katarína Karl’ováa ${ }^{2}$ Azadeh Ghannadan ${ }^{\mathrm{a}}$ \\ ${ }^{a}$ Department of Theoretical Physics and Astrophysics, Faculty of Science, P. J. Šafárik University, Park Angelinum 9, 04001 Košice, Slovakia
}

\begin{abstract}
A spin-1/2 Ising-Heisenberg branched chain constituted by regularly alternating Ising spins and Heisenberg dimers involving an additional side branching is exactly solved in a magnetic field by the transfer-matrix method. The spin$1 / 2$ Ising-Heisenberg branched chain involves two different Ising and one Heisenberg coupling constants. The overall ground-state phase diagram is formed by three different ground states emergent depending on a mutual interplay between the magnetic field and three considered coupling constants: the modulated quantum antiferromagnetic phase, the quantum ferrimagnetic phase, and the classical ferromagnetic phase. It is shown that the interaction anisotropy connected to two different Ising coupling constants substantially influences a breakdown of the intermediate one-half magnetization plateau, which represents a macroscopic manifestation of the quantum ferrimagnetic phase.
\end{abstract}

Keywords: Ising-Heisenberg branched chain, magnetization plateau, interaction anisotropy

\section{Introduction}

Quantum Heisenberg spin chains provide an intriguing platform for an investigation of quantum magnetism using fully rigorous calculation methods being completely free of any uncontrolled approximation [1]. A few exactly solved Ising-Heisenberg and Heisenberg branched spin chains have recently attracted considerable attention, because they may exhibit striking quantum critical points of Kosterlitz-Thouless and Gaussian type [2-4]. Moreover, the Ising-Heisenberg and Heisenberg branched spin chains are not only purely theoretical models, but they are closely related to a few real-world experimental realizations from the family of polymeric coordination compounds [5, 6]. In the present work we will investigate a spin-1/2 IsingHeisenberg branched chain whose magnetic structure is

${ }^{\star}$ This work was supported under the grant Nos. VEGA 1/0531/19 and APVV-16-0186.

${ }^{*}$ Corresponding author

Email address: jozef .strecka@upjs.sk (Jozef Strečka)

Preprint submitted to Journal of Magnetism and Magnetic Materials

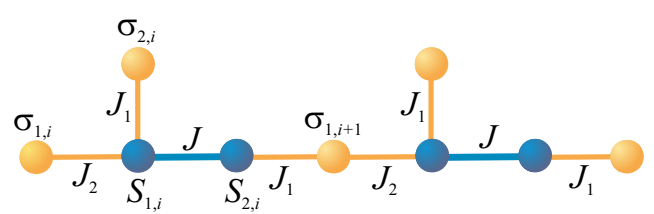

Figure 1: A schematic illustration of the spin-1/2 Ising-Heisenberg branched chain: orange (blue) circles denote lattice positions of the Ising (Heisenberg) spins.

inspired by the heterobimetallic coordination polymer $\left[(\mathrm{Tp})_{2} \mathrm{Fe}_{2}(\mathrm{CN})_{6}\left(\mathrm{OCH}_{3}\right)(\mathrm{bap}) \mathrm{Cu}_{2}\left(\mathrm{CH}_{3} \mathrm{OH}\right) \cdot 2 \mathrm{CH}_{3} \mathrm{OH}\right]$ $(\mathrm{Tp}=$ tris (pyrazolyl)hydroborate, $\mathrm{bapH}=1,3$-bis(amino)2-propanol) [6] to be further abbreviated as $\left[\mathrm{Fe}_{2} \mathrm{Cu}_{2}\right]_{\infty}$.

\section{Model and method}

The spin-1/2 Ising-Heisenberg branched chain, which is inspired by magnetic structure of the heterometallic coordination polymer $\left[\mathrm{Fe}_{2} \mathrm{Cu}_{2}\right]_{\infty}$ originally reported in 
Ref. [6], can be defined through the Hamiltonian:

$$
\begin{aligned}
\hat{\mathcal{H}} & =\sum_{i=1}^{N}\left\{J \hat{\mathbf{S}}_{1, i} \cdot \hat{\mathbf{S}}_{2, i}+J_{1}\left(\hat{S}_{1, i}^{z} \hat{\sigma}_{2, i}^{z}+\hat{S}_{2, i}^{z} \hat{\sigma}_{1, i+1}^{z}\right)\right. \\
& \left.+J_{2} \hat{S}_{1, i}^{z} \hat{\sigma}_{1, i}^{z}-h\left(\hat{S}_{1, i}^{z}+\hat{S}_{2, i}^{z}+\hat{\sigma}_{1, i}^{z}+\hat{\sigma}_{2, i}^{z}\right)\right\} .
\end{aligned}
$$

Here, $\hat{\sigma}_{i}^{z}$ and $\hat{S}_{i}^{\alpha}(\alpha=x, y, z)$ are the spin-1/2 operators ascribed to the Ising and Heisenberg spins, which are schematically shown in Fig. 1 as orange and blue circles, respectively. This schematic illustration additionally involves also notation for three considered coupling constants: the coupling constant $J>0$ stands for the antiferromagnetic Heisenberg exchange interaction within the dimeric units from a backbone of the polymeric chain, while the coupling constants $J_{1}$ and $J_{2}$ correspond to two different Ising-type exchange interactions between the nearest-neighbor Ising and Heisenberg spins. Finally, the Zeeman's term $h$ accounts for a magnetostatic energy of the Ising and Heisenberg spins in a magnetic field, $N$ is the total number of unit cells and the periodic boundary condition $\sigma_{1, N+1} \equiv \sigma_{1,1}$ is imposed for simplicity. For further convenience, the total Hamiltonian (11) can be rewritten as a sum of the cell Hamiltonians $\hat{\mathcal{H}}=\sum_{i=1}^{N} \hat{\mathcal{H}}_{i}$, where each cell Hamiltonian $\hat{\mathcal{H}}_{i}$ is defined by:

$$
\begin{aligned}
\hat{\mathcal{H}}_{i} & =J \hat{\mathbf{S}}_{1, i} \cdot \hat{\mathbf{S}}_{2, i}+J_{1}\left(\hat{S}_{1, i}^{z} \hat{\sigma}_{2, i}^{z}+\hat{S}_{2, i}^{z} \hat{\sigma}_{1, i+1}^{z}\right)+J_{2} \hat{S}_{1, i}^{z} \hat{\sigma}_{1, i}^{z} \\
& -h\left(\hat{S}_{1, i}^{z}+\hat{S}_{2, i}^{z}+\hat{\sigma}_{2, i}^{z}\right)-\frac{h}{2}\left(\hat{\sigma}_{1, i}^{z}+\sigma_{1, i+1}\right) .
\end{aligned}
$$

The cell Hamiltonians $\hat{\mathcal{H}}_{i}$ obviously commute, i.e. $\left[\hat{\mathcal{H}}_{i}, \hat{\mathcal{H}}_{j}\right]=0$, which means that the partition function of the spin-1/2 Ising-Heisenberg branched chain can be partially factorized into the following product:

$$
\mathcal{Z}=\sum_{\left\{\sigma_{1, i}^{z}\right\}} \prod_{i=1}^{N} \sum_{\sigma_{2, i}^{z}} \operatorname{Tr}_{\left[S_{1, i}, S_{2, i}\right]} \mathrm{e}^{-\beta \hat{\mathcal{H}}_{i}}=\sum_{\left\{\sigma_{1, i}^{z}\right\}} \prod_{i=1}^{N} \mathrm{~T}\left(\sigma_{1, i}^{z}, \sigma_{1, i+1}^{z}\right),
$$

where $\beta=1 /\left(k_{\mathrm{B}} T\right), k_{\mathrm{B}}$ is the Boltzmann's factor, $T$ is the absolute temperature, $\operatorname{Tr}_{\left[S_{1, i}, S_{2, i}\right]}$ denotes a trace over degrees of freedom of the Heisenberg dimer from the $i$ th unit cell and $\sum_{\left\{\sigma_{1, i}^{2}\right\}}$ denotes a summation over all possible configurations of the Ising spins from a backbone of the branched chain. The expression $\mathrm{T}\left(\sigma_{1, i}^{z}, \sigma_{1 ; i+1}^{z}\right)=$ $\operatorname{Tr}_{\left[S_{1, i}, S_{2, i}\right]} \mathrm{e}^{-\beta \hat{\mathcal{H}}_{i}}$ is the usual transfer matrix obtained after tracing out spin degrees of freedom of two Heisenberg spins and the Ising spin $\sigma_{2, i}$ from the $i$-th unit cell. To proceed further with the calculations, we have to calculate eigenvalues of the cell Hamiltonian (2) by performing a straightforward diagonalization in the local basis of the Heisenberg spins from the $i$-th unit cell:

$$
\begin{aligned}
& E_{i 1, i 2}=\frac{J}{4} \pm \frac{J_{1}}{2}\left(\sigma_{1, i}^{z}+\sigma_{1, i+1}^{z}\right)+\frac{J_{2}}{2} \sigma_{1, i}^{z} \mp h_{1}, \\
& E_{i 3, i 4}=-\frac{J}{4} \pm \frac{1}{2} \sqrt{\left[\frac{J_{1}}{2}\left(\sigma_{2, i}^{z}+\sigma_{1, i+1}^{z}\right)+\frac{J_{2}}{2} \sigma_{1, i}^{z}\right]^{2}+J^{2}},
\end{aligned}
$$

which should be shifted by the field term $-\frac{h}{2}\left(\sigma_{1, i}^{z}+\sigma_{1, i+1}^{z}\right)-h \sigma_{2, i}^{z}$ accounting for Zeeman's energy of the Ising spins. The corresponding eigenvectors read

$$
\begin{aligned}
& \left|\varphi_{i 1}\right\rangle=|\uparrow\rangle_{1, i}|\uparrow\rangle_{2, i}, \quad\left|\varphi_{i 2}\right\rangle=|\downarrow\rangle_{1, i}|\downarrow\rangle_{2, i}, \\
& \left|\varphi_{i 3}\right\rangle=c_{+}|\uparrow\rangle_{1, i}|\downarrow\rangle_{2, i}+c_{-}|\uparrow\rangle_{1, i}|\downarrow\rangle_{2, i}, \\
& \left|\varphi_{i 4}\right\rangle=c_{+}|\uparrow\rangle_{1, i}|\downarrow\rangle_{2, i}-c_{-}|\uparrow\rangle_{1, i}|\downarrow\rangle_{2, i},
\end{aligned}
$$

where

$$
c_{ \pm}=\frac{1}{\sqrt{2}} \sqrt{1 \pm \frac{J_{1}\left(\sigma_{1, i+1}^{z}-\sigma_{2, i}^{z}\right)+J_{2} \sigma_{1, i}^{z}}{\sqrt{\left[J_{1}\left(\sigma_{1, i+1}^{z}-\sigma_{2, i}^{z}\right)+J_{2} \sigma_{1, i}^{z}\right]^{2}+J^{2}}}} .
$$

In this way one gets an explicit expression for the transfer matrix $\mathrm{T}\left(\sigma_{1, i}^{z}, \sigma_{1 ; i+1}^{z}\right)$ :

$$
\begin{aligned}
\mathrm{T}\left(\sigma_{1, i}^{z},\right. & \left.\sigma_{1 ; i+1}^{z}\right)=\sum_{\sigma_{2, i}^{z}= \pm \frac{1}{2}} \sum_{j=1}^{4} \mathrm{e}^{-\beta E_{j i}}=2 \mathrm{e}^{\frac{\beta h}{2}\left(\sigma_{1, i}^{z}+\sigma_{1, i+1}^{z}\right)-\frac{\beta J}{4}} \\
& \times\left\{\mathrm{e}^{\frac{\beta h}{2}} \cosh \left[\frac{\beta}{2}\left(J_{2} \sigma_{1, i}^{z}+J_{1} \sigma_{1, i+1}^{z}+\frac{J_{1}}{2}+2 h_{1}\right)\right]\right. \\
& +\mathrm{e}^{-\frac{\beta h}{2}} \cosh \left[\frac{\beta}{2}\left(J_{2} \sigma_{1, i}^{z}+J_{1} \sigma_{1, i+1}^{z}-\frac{J_{1}}{2}+2 h_{1}\right)\right] \\
& +\mathrm{e}^{\frac{\beta J}{2}+\frac{\beta h}{2}} \cosh \left[\frac{\beta}{2} \sqrt{\left(J_{2} \sigma_{1, i}^{z}-J_{1} \sigma_{1, i+1}^{z}+\frac{J_{1}}{2}\right)^{2}+J^{2}}\right] \\
& \left.+\mathrm{e}^{\frac{\beta J}{2}-\frac{\beta h}{2}} \cosh \left[\frac{\beta}{2} \sqrt{\left(J_{2} \sigma_{1, i}^{z}-J_{1} \sigma_{1, i+1}^{z}-\frac{J_{1}}{2}\right)^{2}+J^{2}}\right]\right\} .
\end{aligned}
$$

The partition function of the spin-1/2 Ising-Heisenberg 

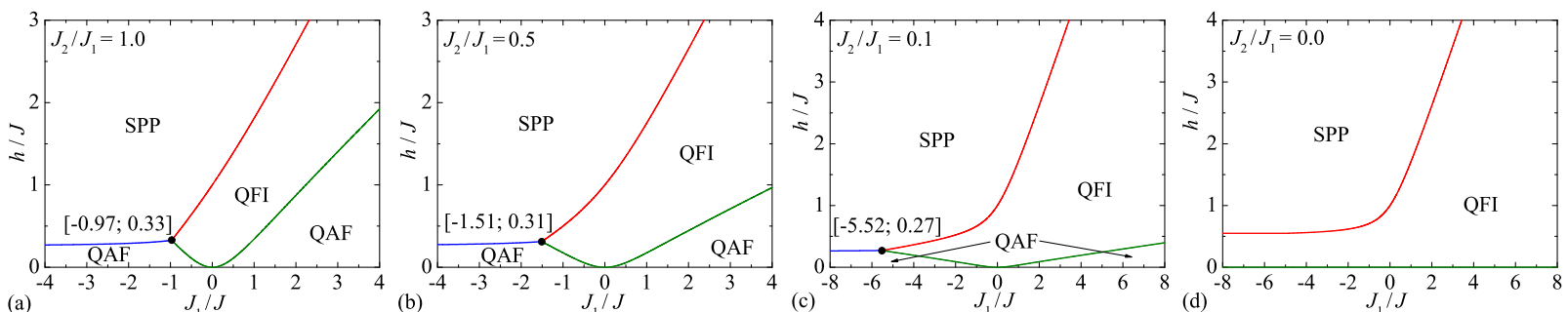

Figure 2: Ground-state phase diagrams of the spin-1/2 Ising-Heisenberg branched chain in the $J_{1} / J-h / J$ plane for four different values of the interaction ratio: (a) $J_{2} / J_{1}=1.0$; (b) $J_{2} / J_{1}=0.5$; (c) $J_{2} / J_{1}=0.1$; (d) $J_{2} / J_{1}=0.0$. The triple-point coordinates are given in square brackets.

branched chain can be expressed in terms of two eigenvalues $\lambda_{+}$and $\lambda_{-}$of the transfer matrix (5):

$$
\mathcal{Z}=\sum_{\left\{\sigma_{1, i}\right\}} \prod_{i=1}^{N} \mathrm{~T}\left(\sigma_{1, i}^{z}, \sigma_{1 ; i+1}^{z}\right)=\operatorname{Tr} \mathrm{T}^{N}=\lambda_{+}^{N}+\lambda_{-}^{N},
$$

which can be written in this compact form:

$$
\lambda_{ \pm}=\frac{1}{2}\left[T_{1}+T_{2} \pm \sqrt{\left(T_{1}-T_{2}\right)^{2}+4 T_{3} T_{4}}\right] .
$$

The parameters $T_{i}(i=1-4)$ mark four elements of twoby-two transfer matrix (5):

$T_{1}=\mathrm{T}(+,+), T_{2}=\mathrm{T}(-,-), T_{3}=\mathrm{T}(+,-), T_{4}=\mathrm{T}(-,+)$, which correspond to four possible states of the Ising spins $\sigma_{1, i}$ and $\sigma_{1, i+1}( \pm$ applies for $\sigma= \pm 1 / 2)$. In thermodynamic limit $N \rightarrow \infty$ the Gibbs free energy can be expressed through larger eigenvalue of the transfer matrix:

$$
G=-k_{\mathrm{B}} T \lim _{N \rightarrow \infty} \frac{1}{N} \ln \mathcal{Z}=-k_{\mathrm{B}} T \ln \lambda_{+} .
$$

Other quantities can be subsequently derived from the Gibbs free energy (8) using standard relations.

\section{Results and discussion}

Let us begin discussion of the most interesting results by a comprehensive analysis of the ground state. It turns out that the spin-1/2 Ising-Heisenberg branched chain may exhibit just three different ground states referred to as the quantum antiferromagnetic phase (QAF):

$$
\begin{aligned}
|\mathrm{Q} A F\rangle & =\prod_{i=1}^{N / 2}\left\{|\uparrow\rangle_{\sigma_{1,2 i-1}}|\uparrow\rangle_{\sigma_{2,2 i-1}}\left(a_{+}|\downarrow, \uparrow\rangle-a_{-}|\uparrow, \downarrow\rangle\right)_{S_{1,2 i-1}, S_{2,2 i-1}}\right. \\
& \left.\otimes|\downarrow\rangle_{\sigma_{1,2 i}}|\downarrow\rangle_{\sigma_{2,2 i}}\left(a_{-}|\downarrow, \uparrow\rangle-a_{+}|\uparrow, \downarrow\rangle\right)_{S_{1,2 i}, S_{2,2 i}}\right\},
\end{aligned}
$$

the quantum ferrimagnetic phase (QFI):

$$
|\mathrm{QFI}\rangle=\prod_{i=1}^{N}|\uparrow\rangle_{\sigma_{1, i}}|\uparrow\rangle_{\sigma_{2, i}}\left(b_{+}|\downarrow, \uparrow\rangle-b_{-}|\uparrow, \downarrow\rangle\right)_{S_{1, i}, S_{2, i}},
$$

and the saturated paramagnetic phase (SPP):

$$
|\mathrm{SPP}\rangle=\prod_{i=1}^{N}|\uparrow\rangle_{\sigma_{1, i}}|\uparrow\rangle_{\sigma_{2, i}}|\uparrow, \uparrow\rangle_{S_{1, i}, S_{2, i}}
$$

For a shorthand notation the QAF and QFI ground states are defined through the probability amplitudes:

$$
a_{ \pm}=\frac{1}{\sqrt{2}} \sqrt{1 \pm \frac{J_{1}+\frac{J_{2}}{2}}{\sqrt{\left(J_{1}+\frac{J_{2}}{2}\right)^{2}+J^{2}}}}
$$

and

$$
b_{ \pm}=\frac{1}{\sqrt{2}} \sqrt{1 \pm \frac{\frac{J_{2}}{2}}{\sqrt{\left(\frac{J_{2}}{2}\right)^{2}+J^{2}}}} .
$$

It is worth mentioning that the QAF ground state with translationally broken symmetry is consistent with existence of zero magnetization plateau in a zero-temperature magnetization curve due to a null total magnetization, while the QFI ground state is responsible for presence of the intermediate one-half plateau if the total magnetization is scaled with respect to its saturation value.

The ground-state phase diagrams of the spin-1/2 IsingHeisenberg branched chain are plotted in Fig. 2] in the $J_{1} / J-h / J$ plane for four selected values of the interaction anisotropy $J_{2} / J_{1}$. It can be concluded that the groundstate phase diagrams are formed regardless of the interaction anisotropy $J_{2} / J_{1}$ by the same three ground states 

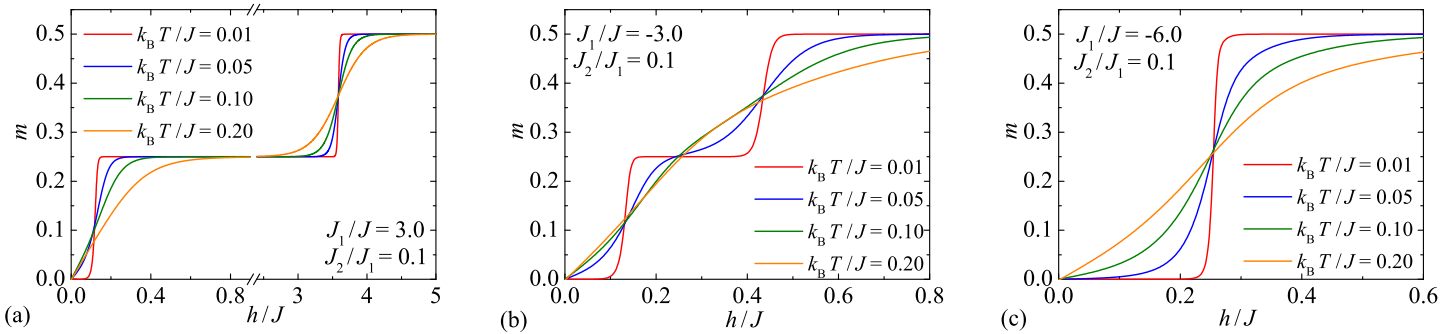

Figure 3: A few typical isothermal magnetization curves of the spin-1/2 Ising-Heisenberg branched chain for the fixed value of the interaction anisotropy $J_{2} / J_{1}=0.1$ and three different values of the interaction ratio: (a) $J_{1} / J=3.0$; (b) $J_{1} / J=-3.0$; (c) $J_{1} / J=-6.0$.

QAF, QFI and SPP as previously reported in Ref. [4] for the isotropic case with $J_{2} / J_{1}=1$, see Fig. 2] a). The interaction anisotropy, i.e. the decline of the interaction ratio from the value $J_{2} / J_{1}=1$, merely causes an extension of the QFI ground state down to lower values of the interaction ratio $J_{1} / J$. On the other hand, the QAF ground state is gradually suppressed by the interaction anisotropy (i.e. when the interaction ratio $J_{2} / J_{1}$ decreases) until the QAF ground state completely disappears from the phase diagram in the limit $J_{2} / J_{1} \rightarrow 0$.

To verify the aforedescribed behavior, a few typical isothermal magnetization curves of the spin-1/2 IsingHeisenberg branched chain are displayed in Fig. 3 for the fixed value of the interaction anisotropy $J_{2} / J_{1}=0.1$ and three selected values of the interaction ratio $J_{1} / J=3.0$, -3.0 and -6.0 , respectively. It can be seen that a relatively wide one-half plateau and narrow zero plateau can be observed by considering the antiferromagnetic Ising coupling $J_{1} / J=3.0$ [see Fig. 3(a)], while the width of zero plateau extends and of one-half plateau shrinks by considering the ferromagnetic Ising coupling $J_{1} / J=-3.0$ [see Fig. 3(b)]. If the ferromagnetic Ising interaction is sufficiently strong one detects a mere existence of zero plateau and a full breakdown of the one-half plateau (see Fig. 3] c) for $J_{1} / J=-6.0$ ). It is noteworthy that the depicted magnetization curves are in a perfect accordance with the established ground-state phase diagrams (c.f. Figs. 2] and 3), whereby the intermediate one-half plateau is absent if a relative strength of the ferromagnetic Ising coupling constant exceeds the particular value ascribed to a triple coexistence point of the QAF, QFI and SPP ground states.

\section{Conclusion}

In the present paper we have exactly solved using the transfer-matrix method the spin-1/2 Ising-Heisenberg branched chain with two different Ising and one Heisenberg coupling constants in a magnetic field. It has been verified that the investigated quantum spin chain may exhibit just three different ground states QAF, QFI and SPP depending on a mutual interplay between the magnetic field and three considered coupling constants. The QAF and QFI ground states with a quantum entanglement between the Heisenberg dimers are responsible for presence of intermediate zero and one-half plateaus in zero- and low-temperature magnetization curves, whereby a relative size of the intermediate magnetization plateaus depends basically on the interaction anisotropy. A full breakdown of the intermediate one-half magnetization plateau has been additionally detected for the particular case with sufficiently strong ferromagnetic Ising coupling constants.

\section{References}

[1] D.C. Mattis, The Many-Body Problem, World Scientific, Singapore, 1993.

[2] L.M. Veríssimo, M.S.S. Pereira, J. Strečka, M.L. Lyra, Phys. Rev. B 99 (2019) 134408

[3] F. Souza, L.M. Veríssimo, J. Strečka, M.L. Lyra, M.S.S. Pereira, Phys. Rev. B 102 (2020) 064414

[4] K. Karl'ová, J. Strečka, M.L. Lyra, Phys. Rev. E 100 (2019) 042127.

[5] H. Wang, L.-F. Zhang, Z.-H. Ni, W.-F. Zhong, L.-J. Tian, J. Jiang, Cryst. Growth Des. 10 (2010) 4231. 
[6] L.-C. Kang, X. Chen, H.-S. Wang, Y.-Z. Li, Y. Song, J.-L. Zuo, X.-Z. You, Inorg. Chem. 49 (2010) 9275. 